\title{
EL PAPEL DEL MODELO DE LA TRIPLE HÉLICE COMO SISTEMA DE INNOVACIÓN PARA AUMENTAR LA RENTABILIDAD EN UNA PYME COMERCIALIZADORA
}

The role of the triple helix model as an innovation system to increase profitability in a SME

\author{
José Antonio Velázquez-Juárez
}

Maestro en Administración, Universidad Autónoma de Querétaro, Querétaro-México, jose_antonio_velazquez@hotmail.com

\section{Luis Rodrigo Valencia-Pérez}

Doctor en Gestión Tecnológica e Innovación, Universidad Autónoma de Querétaro, Querétaro-México, royvalper@hotmail.com

\section{Juan Manuel Peña-Aguilar}

Doctor en Gestión Tecnológica e Innovación, Universidad Autónoma de Querétaro, Querétaro, México, juan_manuelp@hotmail.com

\section{Cómo citar/ How to cite}

Velásquez-Juárez, J., Valencia-Pérez, L. y Peña-Aguilar, J. (2016). El papel del modelo de la triple hélice como sistema de innovación para aumentar la rentabilidad en una Pyme comercializadora. Revista CEA, 2(3), 101-112.

Recibido: 15 de octubre de 2015

Aceptado: 30 de noviembre de 2015

\section{Resumen}

El artículo presenta el impacto a lograr por la implementación de un proceso de transferencia tecnológica a través de un escalamiento tecnológico, con el objetivo de generar innovaciones en procesos e incrementar la rentabilidad de una Pyme comercializadora, utilizando un sistema de innovación llamado modelo de la triple hélice, donde las interacciones entre instituciones públicas y privadas buscan producir, intercambiar, difundir y utilizar nuevos conocimientos $y$ avances tecnológicos.

Como elementos teóricos escogidos de la problemática tratada en el presente artículo, se consideraron temas como el modelo de la triple hélice, transferencia tecnológica, innovación tecnológica y escalamiento tecnológico.

Para evaluar y verificar la viabilidad del proyecto, se incluyó un estudio económico-financiero que valora la rentabilidad a obtener por el desarrollo del proyecto, además de un análisis estadístico con la finalidad de revisar si una disminución en los costos de producción aumenta las utilidades, como consecuencia del proyecto.

Palabras clave: innovación tecnológica, transferencia tecnológica, triple hélice, Pymes, rentabilidad.

\begin{abstract}
The article presents the targeted impact for the implementation of a technology transfer through a technological scaling up with the objective of generating innovations in processes and improving the profitability of a retailing SME by using an innovation system called triple helix model, where interactions between public and private institutions try to produce, interchange, diffuse and use new knowledge and technological advances.

As theoretical elements chosen from the problem addressed in this article, subjects such as triple helix model, transfer of technology,
\end{abstract}


technological innovation and technological scaling up were used.

In order to evaluate and verify the viability of the project, a financial analysis was included to assess the profitability to obtain from the development of the project, which was also supported by a statistical analysis with the purpose of reviewing whether a cost reduction happens as an effect produced by the increase of net income of this project.

Keywords: technologic innovation, technology transfer, triple helix, SME, profitability.

\section{INTRODUCCIÓN}

El desarrollo económico de un país o región depende directamente de las actividades de innovación tecnológica, lo cual representa un impacto directo sobre el rápido crecimiento de diversas economías emergentes y se refleja inmediatamente sobre aquellas naciones que se han visto rezagadas en actividades de innovación tecnológica (OCDE, 2006; Schumpeter, 1934).

Por ello, las condiciones para la creación continua de alta tecnología y el crecimiento económico pueden identificar y localizar a la organización específica. Una forma de realizar esta vinculación es a través del modelo de la Triple Hélice propuesto por Etzkowitz (2000), y consiste en un modelo que permite una vinculación entre disciplinas y conocimientos, donde la universidad tiene un papel estratégico y es la base para generar las relaciones con la empresa.

La aplicación de este sistema de innovación tecnológica puede facilitar que los procesos productivos de las PYMES generen un mayor intercambio de información, adquisición y transmisión de conocimiento, tener mayor acceso a recursos de capital y encontrar mecanismos de cooperación con otras empresas e instituciones que participen en proyectos de innovación tecnológica.
En el presente artículo, se resalta la importancia que tiene la transferencia tecnológica como una estrategia que impulsa la generación de innovaciones de productos y procesos para generar un aumento en la productividad de una empresa comercializadora, a través de un proyecto de trasferencia tecnológica hecho en conjunto con el laboratorio de gestión tecnológica e innovación de la Facultad de Contaduría y Administración de la Universidad Autónoma de Querétaro.

El proceso utilizado para realizar la transferencia tecnológica fue un escalamiento tecnológico, que consistió en adquirir capacidades tecnológicas con base en las demandas hechas por el mercado para mejorar los procesos de transformación de materias primas en productos de forma más eficiente, reorganizando el sistema de producción, destacando la importancia que tienen los diferentes elementos que integran al modelo de la triple hélice sobre la capacidad acumulativa de los procesos de innovación tecnológica.

\section{MARCO TEÓRICO}

\section{Modelo de la triple hélice}

Las relaciones Universidad-Empresa-Estado son altamente requeridas en las economías modernas para el crecimiento y desarrollo de un territorio; los resultados de competitividad brindados por esta triple alianza están demostrados a través de cómo las grandes universidades del mundo reciben recursos de sectores públicos y privados para producir conocimiento de alto nivel, que da como resultado las distintas innovaciones introducidas en diversos sectores empresariales del mundo (Jerome, 2011; Brunet y Baltar, 2010; Fernández, Castro, Conesa, y Gutiérrez, 2000).

Por ello, las condiciones para la creación continua de alta tecnología y el crecimiento económico pueden identificar y localizar a la organización específica. Una forma de realizar esta vinculación es a través del modelo de la triple hélice propuesto por Etzkowitz (2000); 
consiste en un modelo que permite una vinculación entre disciplinas y conocimientos, donde la universidad tiene un papel estratégico y es la base para generar las relaciones con la empresa. Cada una de las hélices genera relaciones internamente, pero también interactúan en términos de intercambio de bienes, servicios y en término de sus funciones. Tanto los roles funcionales como institucionales pueden ser compensados a partir de las expectativas basadas en la generación e intercambio de conocimiento entre los tres elementos (ver Fig. 1).

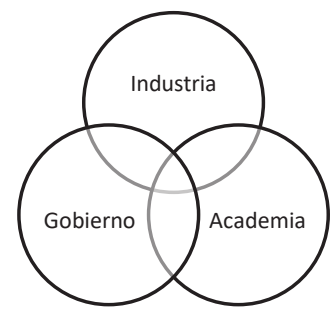

Figura 1. Estructura del modelo triple hélice

Figure 1. Triple helix model

Fuente: elaboración propia a partir de Etzkowitz (2000) y Jerome (2011).

\section{Transferencia tecnológica}

La transferencia tecnológica es un conjunto de mecanismos referente a las ventas 0 concesiones hechas con ánimo lucrativo, de un conjunto de conocimientos que deben permitir al licenciatario o comprador fabricar en las mismas condiciones que el licenciante o vendedor (Escorsa y Valls, 2005).
La transferencia tecnológica debe proporcionar al comprador un conjunto escrito $u$ oral de conocimientos que le permitan utilizar, en las mismas condiciones que el vendedor, un conjunto de herramientas, tecnologías y procedimientos para poder producir en las mismas condiciones que el vendedor y que la producción obtenida pueda convertirse en una operación rentable.

Para ello, se presenta en la Tabla 1, los siguientes mecanismos de transferencia tecnológica y los actores implicados en el proceso de transferencia tecnológica.

Tabla 1. Transferencia de tecnología. Mecanismos y actores

Table 1. Technology transfer. Procedures and actors

\begin{tabular}{|c|c|}
\hline Mecanismos & Actores \\
\hline $\begin{array}{lr}\text { Licencias, } & \text { asistencia } \\
\text { técnica, } & \text { alianzas } \\
\text { estratégicas } & \end{array}$ & $\begin{array}{l}\text { Universidades, Bancos, } \\
\text { Gobiernos, Empresas, } \\
\text { Sociedad }\end{array}$ \\
\hline
\end{tabular}

Fuente: elaboración propia a partir de Escorsa y Valls (2005, p. 269).

Castellanos (2007) explica que la transferencia tecnológica representa un mecanismo de satisfacción de necesidades mucho más complejo, en donde se considera la situación de la tecnología y su uso, de saber si se encuentra en condiciones de realizarlo, el precio que se pagaría por el proceso, la forma de hacerlo y las condiciones en que se encuentra la empresa para ajustar esa nueva tecnología a su realidad.

Dicho proceso conlleva los siguientes elementos (Ver Fig. 2).

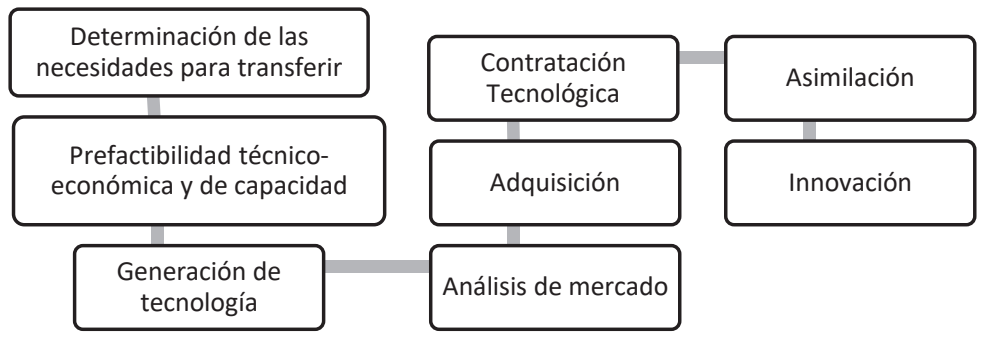

Figura 2. Proceso de transferencia tecnológica

Figure 2. Technology transfer process

Fuente: elaboración propia a partir de Castellanos (2007). 


\section{Innovación tecnológica}

La innovación, se concibe como un conjunto de mecanismos que permiten mejoras en procesos tecnológicos o en formas de hacer un conjunto de actividades, y que se manifiesta principalmente mediante cambios en productos, procesos, nuevos enfoques en mercadotecnia, nuevas formas de distribución e incluso nuevas concepciones de alcance (Porter, 1985; Porter, 1990; Gallouj y Weinstein, 1997; Cegarra, 2004; Friedman, 2008).

Las innovaciones suelen ser más de forma incremental que radicales, ya que el desarrollo de este tipo de innovaciones está muy relacionado con la acumulación de conocimientos, proveniente del aprendizaje organizacional, al igual que el avance que haya en investigación y desarrollo, junto a una fuerte inversión en el desarrollo de habilidades y conocimientos.

Al respecto, Castellanos (2007) explica que la innovación tecnológica y la generación de conocimiento aplicado representa la característica central del sistema económico, que ya está dejando de ser representada por la división del trabajo o por la producción en masa de productos estandarizados.

Castellanos plantea que la innovación aparece como una ráfaga discontinua e irregular en el tiempo, en diferentes sectores de la economía, ya que hay factores como la realización de inversiones, generación de empleos, estímulos a la demanda de capital, bienes de consumo durables, intermedios y finales, que intervienen de manera directa en estos procesos de transformación tecnológica.

\section{Tipos de innovación tecnológica}

Davenport, Leibold y Voelpel (2006) explican que existe una amplia y a menudo confusa clasificación sobre innovación, que está todavía en las progresiones mecánicas y lógicas de la economía industrial. En la Tabla 2, se muestra la clasificación sobre los diversos enfoques de la innovación, ilustrando su multidimensionalidad y variedad de opiniones.

Tabla 2. Clasificación de Innovaciones tecnológicas

Table 2. Technological innovations category

\begin{tabular}{|c|c|}
\hline $\begin{array}{l}\text { Innovación } \\
\text { disruptiva }\end{array}$ & $\begin{array}{l}\text { Recibe mucha atención, ya que los } \\
\text { mercados aparecen de la nada y } \\
\text { crean nuevas fuentes masivas de } \\
\text { ganancias, provenientes } \\
\text { principalmente de discontinuidades } \\
\text { tecnológicas }\end{array}$ \\
\hline $\begin{array}{c}\text { Innovación } \\
\text { aplicada }\end{array}$ & $\begin{array}{c}\text { Toma tecnologías existentes hacia } \\
\text { nuevos mercados para aplicarse en } \\
\text { nuevos propósitos y generar nuevos } \\
\text { usos en productos y servicios }\end{array}$ \\
\hline $\begin{array}{c}\text { Innovación } \\
\text { en } \\
\text { productos }\end{array}$ & $\begin{array}{l}\text { Toma productos disponibles en } \\
\text { mercados establecidos y los lleva a } \\
\text { un nivel más alto, enfocándose en } \\
\text { mejorar su uso, reducir costos de } \\
\text { producción o aumento en su } \\
\text { desempeño }\end{array}$ \\
\hline $\begin{array}{c}\text { Innovación } \\
\text { en } \\
\text { procesos }\end{array}$ & $\begin{array}{c}\text { Toma procesos para productos } \\
\text { disponibles en mercados } \\
\text { establecidos con la intención de } \\
\text { volverlos más eficientes o efectivos }\end{array}$ \\
\hline
\end{tabular}

Al respecto, Ahmed et al. (2012) señalan que la innovación puede presentarse en distintos formatos o tipos, tales como la innovación estratégica, innovación en productos y en procesos, que se derivan de la variedad de significados adscritos, pero en términos generales, se identifica a partir de la capacidad de la empresa para poder controlarlos o que se encuentran fuera de su campo de influencia. A continuación, se presentan los tipos de innovación, tomando en cuenta la capacidad de control de la empresa para su clasificación (ver Fig. 3). 


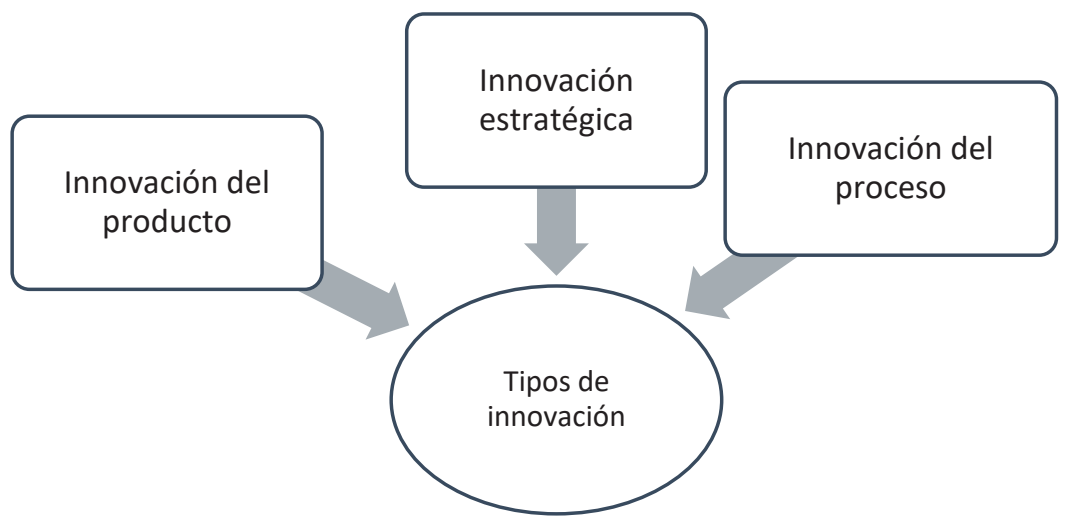

Figura 3. Tipos de innovación tecnológica

Figure 3. Technological innovations types

Fuente: elaboración propia a partir de Ahmed et al. (2012, p.7).

\section{Escalamiento tecnológico}

El escalamiento tecnológico representa una oportunidad de realizar múltiples cambios para la empresa, ya que se plantea la posibilidad de pasar de procesos productivos sencillos a procesos productivos complejos en una organización, que normalmente está acompañada de utilizar mecanismos para la generación y acumulación de capacidades tecnológicas y está dirigido a realizar mejoras en la calidad y hacer modificaciones en el diseño de un producto, sin alterar de manera intrínseca su composición (Dieter, 1986).
Por otro lado, Gereffi y Tam (1999) explican que el escalamiento industrial es un concepto que implica múltiples cambios en los procesos productivos que facilite la producción de bienes y servicios, con la finalidad de alcanzar un mayor margen de rentabilidad y cambios a lo largo de la cadena de valor de una organización.

Gereffi y Tam añaden que el escalamiento tecnológico cuenta con cuatro niveles de impacto del escalamiento industrial, que considerados del ámbito más general al más específico se podrían listar de la siguiente manera (ver Fig. 4).

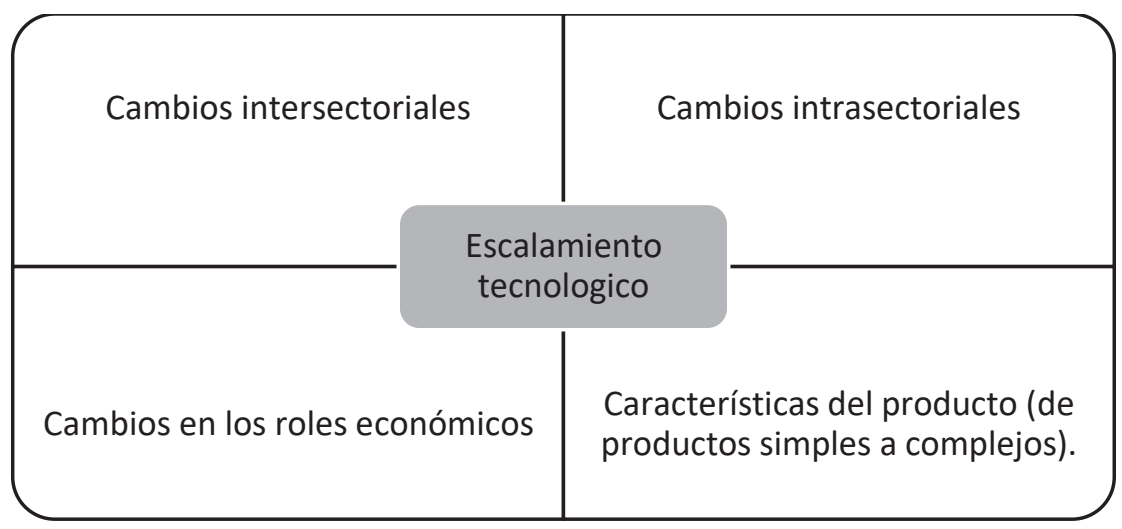

Figura 4. Tipos de escalamiento industrial

Figure 4. Industrial upgrading types

Fuente: elaboración propia a partir de Gereffi y Tam (1999, p.2). 


\section{METOdOLOGÍA}

\section{Diseño de la investigación}

Se utilizará como método de investigación, el estudio de caso, ya que puede ser abordado utilizando información cuantitativa y cualitativa, para poder eventualmente indagar de manera detallada, comprensiva, sistemática y de manera profunda el caso objeto de interés (Flick, 2012).

El presente artículo se encuentra inserto dentro del paradigma cuantitativo, con un tipo de estudio correlacional, descriptivo y con profundidad transversal en el tiempo. En primer lugar, se utilizó un estudio descriptivo con la finalidad de especificar las propiedades, las características, del objeto de estudio sometido al presente análisis. Además, se consideró pertinente realizar un estudio correlacional con la finalidad de medir el grado de relación existente entre las variables de estudio seleccionadas. (Bernal, 2011).

Como complemento de la presente investigación, se utilizará a manera de simulación el impacto que se espera lograr con el proceso de implementación del escalamiento tecnológico en el desempeño financiero estimado del proyecto, a partir de pronósticos e indicadores, para poder conocer los resultados a lograr como consecuencia del cambio en el proceso de escalamiento tecnológico y productivo, complementado con un análisis estadístico para mostrar cómo el proceso de expansión de capacidades puede influir en el aumento de las utilidades y en la reducción de los costos de producción.

\section{Descripción del estudio de caso}

El presente caso de estudio se enfoca en estudiar el proceso de innovación tecnológica en una Pyme comercializadora del sector alimenticio, a través de mecanismos de transferencia de tecnología, información y conocimientos, conocido como modelo de la triple hélice, con la finalidad de realizar interacciones que incentiven el intercambio de recursos financieros, materiales y humanos para alcanzar mayores niveles de desarrollo tecnológico y económico.

En la actualidad, este tipo de organizaciones en México son la base de la economía, ya que representan el $\mathbf{9 7 \%}$ del total de las empresas, generan el $79 \%$ de empleos y aportan el $23 \%$ del Producto Interno Bruto (PIB) (Chávez et al., 2014).

Cabe destacar que la problemática que viven las Pyme en México, se considera multifactorial y compleja, en donde las principales manifestaciones de dichas problemáticas se pueden empezar a observar desde el momento en que los niveles de inversión para tecnología, infraestructura, seguridad, entre otras, son del orden de $0.5 \%$ al $0.7 \%$ aproximación; situación que ha dado lugar al estancamiento prolongado con un resultado prácticamente nulo de su crecimiento económico.

A partir de la problemática mencionada sobre el papel de los sistemas de innovación de la transferencia tecnológica y su influencia en los resultados potenciales originados por la implementación de innovaciones tecnológicas, se plantea la siguiente pregunta: ¿qué efecto pueden tener las interacciones generadas por sistemas de innovación, como el modelo de la triple hélice, sobre la rentabilidad de una PYME.

\section{Objetivos}

\section{Objetivo General}

Explicar el impacto que puede generar un proyecto de transferencia tecnológica aplicado a la generación de innovaciones en procesos para el aumento de la rentabilidad de una Pyme comercializadora mediante el uso de sistemas de innovación. 


\section{Objetivos Específicos}

Describir los cambios que puede generar el proceso de transferencia tecnológica en los procesos productivos de la organización.

Determinar de manera económica y financiera el efecto que puede generar el proceso de innovación tecnológica sobre las ventas y las utilidades relacionadas al proyecto.

\section{RESULTADOS}

Se busca con el artículo mostrar los resultados potenciales del proceso de escalamiento tecnológico, que permitirían generar una innovación incremental dentro de una empresa comercializadora del sector alimenticio, asociada a la incorporación de tecnologías de proceso, mediante automatizaciones progresivas $y$ control de la calidad en cada una de las etapas del proceso y mostrando modificaciones en el diseño del producto, para volverlo más llamativo y práctico para el mercado meta, sin que esto represente una modificación en las características intrínsecas del producto.

Es necesario mencionar que el proyecto actual descrito en el estudio aún no se encuentra en operación, sin embargo, se desarrollará en el transcurso del presente año como una condición para la obtención del financiamiento otorgado por el Gobierno Federal, como parte de las cualidades que tiene el modelo de triple hélice enfoque de estudio seleccionado para la presente investigación.

Para ello, se muestra las actividades necesarias para la identificación de problemas que impulsaron el desarrollo del proyecto de innovación tecnológica (ver Fig. 5).

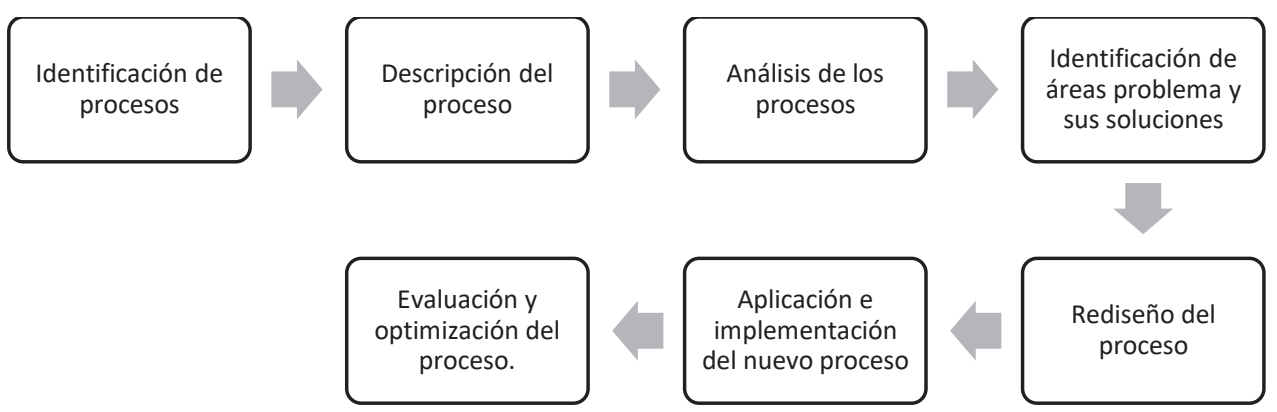

Figura 5. Identificación de problemáticas en el proceso de la línea de moldeado

Figure 5. Identification of problem in process of molding line

Fuente: elaboración Propia

\section{Análisis financiero}

Con la finalidad de tener una mayor certidumbre y una reducción sobre el riesgo operativo y tecnológico que puedan provenir del proyecto de innovación tecnológica, se llevó a cabo una estimación del desempeño financiero del proyecto, para saber la viabilidad económica del proceso de escalamiento tecnológico, donde se mostrarán dos flujos de efectivo: el primero describirá los resultados del proyecto antes de llevar a cabo el escalamiento; y el segundo flujo mostrará los resultados económicos a lograr al realizar el escalamiento tecnológico de los procesos de fabricación.

El diseño del estudio económico se hizo con base en las aportaciones de Serrano (2011) Sapag (2011) y Baca (2013), donde se busca principalmente tener mayores argumentos para poder aceptar o rechazar el proyecto, dado el valor que puedan tener los ingresos que genere la iniciativa de innovación tecnológica a lo largo 
del tiempo. A continuación, se muestra una estructura general del análisis económico, donde se tomaron datos de la inversión inicial que son base para calcular depreciaciones y amortizaciones anuales; es además un dato que se utiliza en el balance general. Además, se requiere determinar los ingresos que ocasionaría la innovación y de sus costos totales, con la finalidad de elaborar los estados financieros proyectas, incluido el flujo de efectivo, y realizar la evaluación económica (financiera). (Ver Fig. 6).

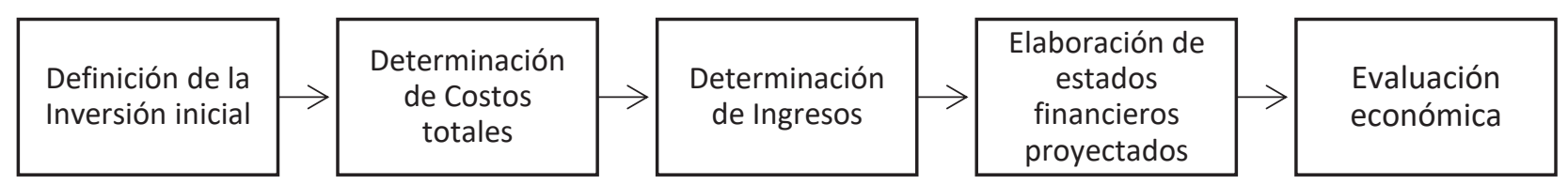

Figura 6. Estructura del análisis económico-financiero

Figure 6. Economic and financial analysis

Fuente: elaboración propia a partir de Baca (2013) y Sapag (2011).

El análisis financiero se efectúa con una tasa de rendimiento mínimo requerida del $10.46 \%$, esto es, un porcentaje de las ganancias que se esperan obtener por el desarrollo del proyecto. Para ello, en la Tabla 4 se presenta los criterios que conformaron a la tasa de rendimiento requerido para el proyecto y que permite fijar la tasa con la que el proyecto es aceptable, a partir de una inversión en un análisis de flujo de efectivo descontado.

Tabla 3. Criterios para la fijación de la tasa de rendimiento mínimamente aceptable

Table 3. Minimally acceptable criteria for setting the

\begin{tabular}{|c|c|}
\hline \multicolumn{2}{|c}{ rate of return } \\
\hline Criterio & $\begin{array}{c}\text { Valor actualizado al mes } \\
\text { de abril de } 2016\end{array}$ \\
\hline CETES & $3.74 \%$ \\
\hline Inflación & $2.54 \%$ \\
\hline $\begin{array}{c}\text { Tasa de Interés } \\
\text { interbancaria de } \\
\text { equilibrio }\end{array}$ & $4.18 \%$ \\
\hline
\end{tabular}

Fuente: elaboración propia a partir de Baca (2013).
Es de suma importancia mencionar que para la definición de la tasa de rendimiento mínimamente aceptada para el proyecto, no se consideró la tasa de interés a pagar por algún financiamiento bancario, puesto que el financiamiento provino principalmente del apoyo brindado por el Gobierno federal, la cual no implica el pago de algún interés por el uso del dinero prestado, es decir, se van a fondo perdido y debido a esta razón, la tasa de rendimiento mínimamente aceptada para este proyecto es muy baja.

Sin embargo, para la aplicación de otros proyectos puede considerarse involucrar este criterio, pero se tendría que lograr mayores flujos de efectivo. Por ello, en la Tabla 5, se muestra un análisis comparativo entre los resultados financieros que se obtenían por parte de la empresa antes de realizar el proyecto de innovación tecnológica, y además se incluye una comparación de los métodos de evaluación económica utilizados como el Valor Presente Neto (VPN) y Tasa Interna de Retorno (TIR), además de hacer una interpretación de estas técnicas y las decisiones que se pueden tomar a partir de los criterios de aceptación de proyectos establecidos por Baca (2013). 
Tabla 5. Proyecciones económico-financiero del proyecto

Table 5. Economic and financial projections of the project

\begin{tabular}{|c|c|c|}
\hline Periodo & 2010-2014 & 2015-2019 \\
\hline Situación & $\begin{array}{c}\text { Antes del } \\
\text { Escalamiento } \\
\text { Tecnológico }\end{array}$ & $\begin{array}{l}\text { Después del } \\
\text { escalamiento } \\
\text { tecnológico }\end{array}$ \\
\hline Ingresos & $\$ 119,655,000$ & $\$ 169,743,420$ \\
\hline $\begin{array}{l}\text { Costos } \\
\text { Totales }\end{array}$ & $\$ 71,832,508$ & $\$ 65,440,290$ \\
\hline $\begin{array}{l}\text { Utilidad antes } \\
\text { de impuestos }\end{array}$ & $\$ 47,822,492$ & $\$ 104,303,130$ \\
\hline $\begin{array}{l}\text { Impuestos } \\
(46 \%)\end{array}$ & $\$ 21,998,346$ & $\$ 47,979,440$ \\
\hline $\begin{array}{l}\text { Utilidad } \\
\text { después de } \\
\text { impuestos }\end{array}$ & $\$ 25,824,146$ & $\$ 56,323,690$ \\
\hline Depreciación & $\$ 10,307,539$ & $\$ 10,307,539$ \\
\hline Utilidad neta & $\$ 36,131,685$ & $\$ 66,631,229$ \\
\hline VPN & $\$ 2,002,704$ & $\$ 6,405,943$ \\
\hline TIR & $13 \%$ & $19 \%$ \\
\hline
\end{tabular}

Fuente: elaboración propia a partir de Baca (2013) y Sapag (2011).

\section{Análisis de los resultados}

En el periodo 2010-2014, antes de llevar a cabo el proceso de escalamiento tecnológico, la mayor parte del proceso de producción y empaquetado se hacía de manera manual. Se logró un total de utilidades de $\$ 36131685$, con un VPN de \$2002704 y una TIR de 13\%.

Por otro lado, una vez hecho el escalamiento tecnológico para el proceso de producción que se proyecta lograr para el periodo 2015-2019, se estima aumentar las utilidades netas a $\$ 66,631,229$ incrementando el VPN a \$6405943, es decir, se espera lograr un aumento muy importante a comparación del periodo previo, antes de llevar a cabo el escalamiento tecnológico.

Al utilizar el criterio de aceptación de proyectos para VPN (Valor Presente Neto) es pertinente la realización del proyecto, ya que el $V P N>0$ $(\$ 6,405,943)$. Asimismo, utilizando el criterio de aceptación de proyectos para la TIR (Tasa Interna de Retorno), se puede llevar a cabo el proyecto, ya que la TIR a obtener con la implementación del escalamiento tecnológico (19\%) es mayor a la tasa de descuento demandada por el proyecto (10.5\%)

\section{Análisis estadístico}

El análisis financiero es complementado mediante un análisis estadístico, utilizando como herramienta la regresión lineal simple, para mostrar cómo a través del escalamiento tecnológico se aumentan las utilidades netas, mediante la reducción de costos de producción, como un beneficio a lograr del proceso de innovación tecnológica.

Para la elaboración del análisis estadístico, se eligió el software denominado Minitab 17, ya que facilita el tratamiento de los datos para la definición de las variables, la elaboración de los gráficos que ayudan a explicar la relación de las tendencias existentes de las variables elegidas para el estudio, además de que la utilización de este programa facilita la interpretación y selección de los datos. En este orden de ideas, se eligió como método de análisis estadístico la regresión lineal simple, ya que se buscó explicar y estudiar la relación que existe entre dos variables: una variable dependiente o explicada (y), sobre una variable independiente o explicativa (x) (Díaz Mata, 2013; Salvatore, 2007), donde se buscó estudiar la relación entre la reducción de costos y el aumento de utilidades o rentabilidad como efecto de haber realizado el proceso de escalamiento tecnológico (Ver fig. 7). 


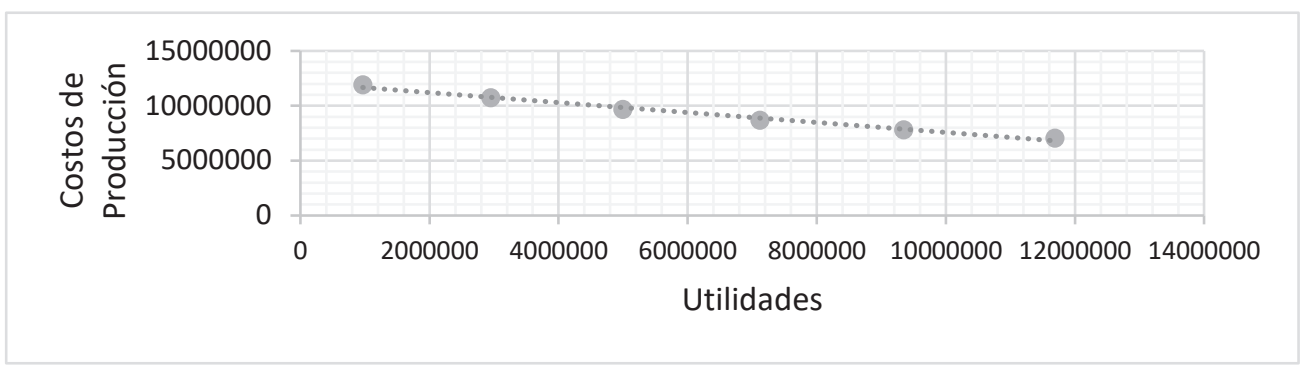

Figura 7. Diagrama de dispersión entre utilidad y costos de producción

Figure 7. Dispersion diagram between utility versus production costs

Fuente: elaboración propia

Con la elaboración del análisis de regresión simple se buscó comprobar el objetivo que se siguió con el proceso de escalamiento tecnológico, reducir costos de producción, reordenando el proceso de fabricación y utilizando de manera eficiente la materia prima, y su relación con el aumento de las utilidades.

Tabla 6. Análisis de regresión lineal y cálculo de coeficiente de correlación

Table 6. Regression analysis and correlation coefficient

\begin{tabular}{lr}
\hline \multicolumn{2}{c}{ Estadísticas de la regresión } \\
\hline Coeficiente de correlación múltiple & 0.99421649 \\
Coeficiente de determinación $\mathrm{R}^{\wedge} 2$ & 0.98846643 \\
$\mathrm{R}^{\wedge} 2$ ajustado & 0.98558304 \\
Error típico & 219442.396 \\
Observaciones & 6 \\
\hline
\end{tabular}

Fuente: elaboración propia

Por lo tanto, en la Tabla 6, se presentan los resultados del análisis de regresión, donde se obtuvo un coeficiente de correlación múltiple de 99.42\%. Posteriormente, se obtuvo un coeficiente de determinación $\left(r^{2}\right)$ de $98.84 \%$, el cual muestra que los puntos quedan cerca del modelo lineal ajustado, originado del valor obtenido, ya que entre más alto el valor, las predicciones son más confiables. Lo anterior indica una fuerte asociación entre estas dos variables, utilidades y reducción de costos, indicando un impacto positivo.

\section{CONCLUSIONES}

Es necesario recalcar que para desarrollar procesos de innovación tecnológica se requiere inicialmente identificar la oportunidad de realizar éstas, por medio de la generación y la formulación de ideas, como solución un problema, el cual se desarrollará para, eventualmente, utilizarse y difundirse. En ello, de la vinculación entre las instituciones de educación, el Gobierno y el sector empresarial, elementos de la triple hélice, resulta una estrategia que se basa en aportar recursos monetarios, humanos y materiales, para que se lleven a cabo actividades de innovación tecnológica, contribuyendo en el mejoramiento de los niveles de productividad y rentabilidad de la empresa, y con ello, al crecimiento económico de una región y país.

Esta situación planteada ha originado que empresas de diferentes sectores e industrias, en especial las de tamaño pequeño y mediano, empiecen con la búsqueda de esquemas para tratar de acceder, asimilar y explotar tecnologías con la finalidad de que se facilite la integración de la tecnología al aparato productivo de una organización.

Respecto al estudio de caso elegido para este artículo, el escalamiento y optimización de la línea de moldeado, mediante un proceso de mejora continua, se pudo analizar los procesos de la empresa que estuvieran relacionadas con esta línea, para poder identificar los cuellos de botella del proceso y localizar oportunidades de 
mejora. Adicionalmente, los resultados de la regresión lineal indican una fuerte asociación entre el aumento de la rentabilidad y la reducción de costos obtenida de la innovación tecnológica.

Sin embargo, al momento de evaluar la efectividad de estos procesos de innovación tecnológica, se requiere medir la relación de la innovación con las ventas de la empresa, el impacto de la innovación sobre la oferta y cartera de productos de la empresa y, de ser necesario, la relación existente entre innovación y actividades de aprendizaje y acumulación de conocimientos. Asimismo, el desarrollo de las innovaciones tecnológicas puede llegar a afectar negativamente los resultados esperados, ya que, por lo general, la nueva tecnología exige unas capacidades diferentes de las que tradicionalmente poseían y es posible que no se cuenten con los recursos necesarios para explotar con éxito la nueva tecnología.

Adicionalmente, el cambio tecnológico puede afectar de distintas maneras al rendimiento de las empresas, como consecuencia de las diferencias en las estrategias relacionadas con factores como la adquisición de la tecnología, la colaboración entre empresas y las estrategias de mercadotecnia. Dicho progreso tecnológico en una organización deberá estar acompañado de una gestión adecuada del conocimiento, ya que es muy común tratar de obtener recursos y copiar capacidades difíciles de imitar, que sean valiosos para lograr ventajas competitivas sostenibles, provocando que los progresos en la competitividad sean fruto de la casualidad o que tengan una duración muy corta.

\section{REFERENCIAS}

Ahmed, P., Shepherd, C. D., Ramos, L. y Ramos, C. (2012). Administración de la innovación. México: Pearson Educación.

Baca, G. (2013). Evaluación de proyectos. México: McGraw Hill.
Bernal, C. A. (2011). Metodología de la Investigación. Bogotá: Pearson.

Brunet, I. y Baltar, F. (2010). Creación de empresas: innovación e instituciones. Madrid: Rama.

Castellanos, O. (2007). Gestión Tecnológica. Medellín: Universidad Nacional de Colombia.

Cegarra, J. (2004). Metodología de la investigación científica y tecnológica. Madrid: ISE.

Chavez, Y. S. (2014). Gestión y Formación de Pymes Tecnológicas. México: Éxodo.

Davenport, T., Leibold, M. and Voelpel ,S. (2006). Strategic Management in the Innovation Economy: Strategic Approaches and Tools for Dynamic Innovation Capabilities. USA: Wiley.

Díaz M., A. (2013). Estadística Aplicada a la administración y economía. México: McGraw Hill.

Dieter, E. (1986). La automatización basada en el uso de computadoras y la internacionalización de la industria electrónica. Implicaciones estratégicas para los países en desarrollo. Berlín: en Mintan (coord.) Industria y nuevas estrategias de desarrollo en América Latina, CIDE, México.

Escorsa, P. Y Valls, J. (2005). Tecnología e innovación en la empresa. Barcelona: Alfaomega.

Etzkowitz, H. Y Leydesdorff, L. (2000). The dynamics of innovation: from National Systems and "Mode 2" to a Triple Helix of university-industry-government relations. Elsevier, 109-123. 
Fernández, I., Castro, E., Conesa, F., y Gutiérrez, A. (2000). Las relaciones universidad empresa: Entre la transferencia de resultados y el aprendizaje regional. Espacios, 21 (2). Recuperado de http://www.revistaespacios.com/a00v21n0 2/60002102.html

Flick, U. (2012). El diseño de la investigación cualitativa. Madrid: Morata.

Friedman, R. (2008). Technology and Innovation Management. New York: Information Science Reference.

Gallouj, F. y Weinstein, O. (1997). Innovation in Services. Research Policy, 537-556.

Gereffi, G. y Tam, T.(1999). Industrial Upgrading and Organization Chains. Instituto of Development Studies, Sussex Universitos.https://www.ids.ac.uk/IDS/globa I/conf/pdfs/iuoc.pdf

INEGI. (2011). Micro, pequeña y gran empresa: estratificación de los establecimientos. México: INEGI.

Jerome, L. W. (2011). Triple Helix Knowledge Clusters. En M. Saad \& G. Zawdie, Theory and Practice of the Triple Helix Systems in
Developing Countries (pág. 471). New York: Routledge.

OCDE. (2006). Guía para la recogida e interpretación de datos sobre innovación. Madrid: Grupo Tragsa.

Porter, M. (1985). Competitive Advantage: Creating and sustaining superior performance. New York: Macmillian.

Porter, M. (1990). The Competitive Advantage of Nations. New York: Harvard Business Review.

Salvatore, D. (2007). Econometría. México: Mcgraw-hill.

Sapag, N. (2011). Proyectos de Inversión: Formulación y Evaluación. Santiago: Pearson.

Schumpeter, J. (1934). The Theory of Economic Development: An Inquiry into Profits, Capital, Credit, Interest, and the Business Cycle. New Jersey: Transaction Publishers.

Serrano Rodríguez, J. (2011). Matemáticas financieras y evaluación de proyectos. Bogotá: Alfaomega. 